Aim of the study: Accurate skin dose assessment is necessary during breast radiotherapy to assure that the skin dose is below the tolerance level and is sufficient to prevent tumour recurrence. The aim of the current study is to measure the skin dose and to evaluate the geometrical/anatomical parameters that affect it.

Material and methods: Forty patients were simulated by TIGRT treatment planning system and treated with two tangential fields of $6 \mathrm{MV}$ photon beam. Wedge filters were used to homogenise dose distribution for 11 patients. Skin dose was measured by thermoluminescent dosimeters (TLD100) and the effects of beam incident angle, thickness of irradiated region, and beam entry separation on the skin dose were analysed.

Results: Average skin dose in treatment course of $50 \mathrm{~Gy}$ to the clinical target volume (CTV) was 36.65 Gy. The corresponding dose values for patients who were treated with and without wedge filter were 35.65 and 37.20 Gy, respectively. It was determined that the beam angle affected the average skin dose while the thickness of the irradiated region and the beam entry separation did not affect dose. Since the skin dose measured in this study was lower than the amount required to prevent tumour recurrence, application of bolus material in part of the treatment course is suggested for post-mastectomy advanced breast radiotherapy. It is more important when wedge filters are applied to homogenize dose distribution.

Key words: breast cancer, conformal radiotherapy, skin dose, in vivo dosimetry.

Contemp Oncol (Pozn) 2016; 20: 137-140 DOI: $10.5114 /$ wo.2015.54396

\section{In vivo skin dose measurement in breast conformal radiotherapy}

\author{
Shokouhozaman Soleymanifard ${ }^{1,2,3}$, Seyed Amir Aledavood ${ }^{4,5}$, \\ Atefeh Vejdani Noghreiyan ${ }^{2}$, Mahdi Ghorbani ${ }^{1}$, Farideh Jamali ${ }^{3}$, \\ David Davenport ${ }^{6}$
}

${ }^{1}$ Medical Physics Research Centre, Mashhad University of Medical
Sciences, Mashhad, Iran

${ }^{2}$ Department of Medical Physics, Faculty of Medicine, Mashhad University of Medical Sciences, Mashhad, Iran

${ }^{3}$ Medical Physics Department, Omid Hospital, Mashhad University of Medical Sciences, Mashhad, Iran

${ }^{4}$ Cancer Research Centre, Faculty of Medicine, Mashhad University of Medical Sciences, Mashhad, Iran

${ }^{5}$ Department of Radiation Oncology, Faculty of Medicine, Mashhad University of Medical Sciences, Mashhad, Iran

${ }^{6}$ Comprehensive Cancer Centres of Nevada, Las Vegas, Nevada, USA

\section{Introduction}

Accurate skin dose assessment is necessary during breast radiotherapy to assure that the skin dose is below the tolerance level and is sufficient to prevent tumour recurrence. Conventionally, breast radiotherapy is performed by photon beams and is frequently boosted with electron beams. For this purpose, two opposed tangential fields are planned to irradiate the tumour bed while sparing normal tissues. Skin dose is mainly originated from the electron contamination existing in the photon beam and also partially from the backscattered photons originating from the underlying tissue layers. Skin dose varies considerably over the surface of the chest wall and depends on a number of factors, including: beam angle, field size, modifying devices in the beam path [1], tangential beam entry separation (breast separation through the central axis) [1, 2], and the treatment techniques used [3].

Due to the skin dose complexity for two opposed tangential beams, some studies have been designed to assess the breast skin dose in experimental [3-5] or in vivo situations [6, 7]. Hsu et al. [8] showed that the skin dose was within 3\% difference for intensity-modulated radiotherapy (IMRT) and conventional techniques. They also studied the effect of bolus material, and their results revealed that the bolus effect was more crucial for entrance dose. In some other studies, the impacts of breast size and beam angle have also been investigated [4, 7].

In our home hospital no wedge filter is usually used for patients who are candidates for post-mastectomy breast radiotherapy. In these cases the dose received by the shallow tissue layers is more than that received by the deeper ones. Therefore, it is usually supposed that the skin dose is sufficient to prevent tumour recurrence, and consequently no bolus material is used to increase the skin dose, except for advanced tumours (stages III-VI). For patients who undergo partial mastectomy or breast conservative operation, wedge filters are mostly used to compensate the dose inhomogeneity in a relatively large tissue volume. The same procedure is performed for patients who have undergone mastectomy but have used a tissue expander. In all cases it is important to prevent skin dose toxicity, whereas for the mastectomy patients the adequacy of skin dose is also important. The present study was designed to measure the skin dose of patients who underwent breast radiotherapy with and without wedge filter. The effect of beam incident angle, the thickness of irradiated region (the thickness of the chest wall), and beam entry separation on skin dose were also analysed. 


\section{Material and methods}

\section{Treatment planning of patients}

A total of 40 breast cancer patients who needed postoperative radiotherapy were selected randomly. Among these patients, 34 patients had been previously treated with a mastectomy surgery (25 cases with stages III or more; 9 cases with stages I or II), and six patients with stages I or II had breast conservative surgery. All of the latter six cases and five cases of patients who had a mastectomy surgery were treated in the presence of a wedge filter to homogenise the dose distribution in relatively thick or curved tissue. Three patients with mastectomy surgery had used tissue expanders. A computed tomography (CT) scanner (Siemens, Somatom Duo Emotion, Germany) equipped with laser and TIGRT treatment-planning system (Linatech, USA) was used to perform patient virtual simulation. During CT scanning patients were positioned supine on a breast board, and the board angle was tailored based on each patient's anatomy. Clinical target volume (CTV), planning target volume (PTV), heart, lungs, and spinal cord were contoured on the CT slices. Two tangential opposed fields were planned, and their isocentres were located in the chest wall. The beam angles were optimised to decrease the heart and lung volume irradiation. In most cases treatment of nodes in the supraclavicular region was necessary (36 patients); therefore, the supraclavicular and the axillary nodes were contoured and an anterior field was planned to irradiate the region. The anterior field was tilted by $5-10$ degrees to spare the spinal cord. The patient's couch was also tilted to prevent the overlapping of the tangential and supraclavicular fields. Tangential fields were designed to deliver the prescribed dose in at least $90 \%$ volume of the CTV and were justified based on dose homogeneity, dose volume histogram (DVH) of planning target volume, and DVH of the organs at risk. The nominal prescribed dose was 50.0 Gy in twenty-five fractions using 6-MV photon beams (Varian accelerator, Clinac 600).

\section{Skin dose measurement}

Lithium fluoride thermoluminescent dosimeters (TLDs) with cross section of $3 \times 3 \mathrm{~mm}^{2}$ and thickness of $0.9 \mathrm{~mm}$ (TLD-100, Harshaw-Bicron, Cleveland, OH, USA) were used

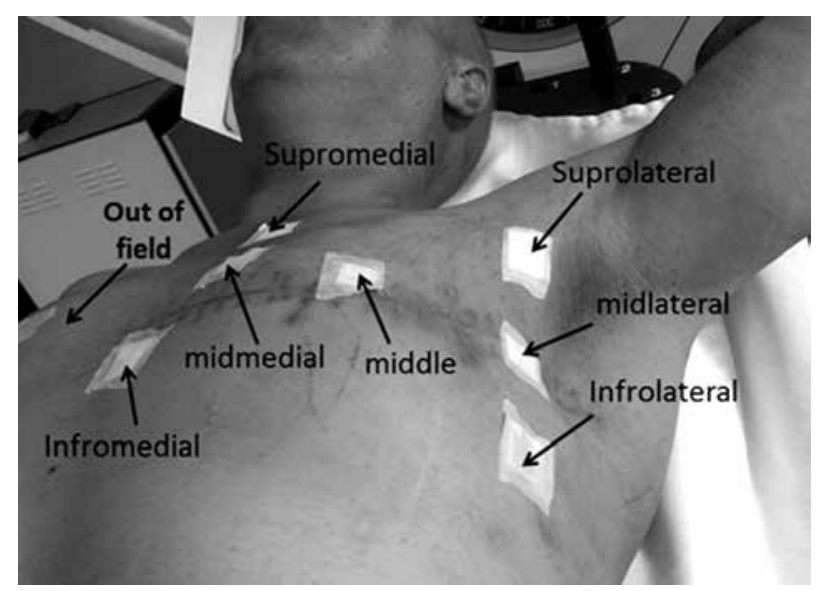

Fig. 1. Positions of the TLD chips on the skin to measure the skin dose. A 3500 TLD reader (HarshowBicron, USA) was used to read the TLDs. TLD chips were calibrated as described in a previous work [9]. Briefly, 15 TLD chips (calibration dosimeters) were irradiated to determine their element correction coefficients (ECCS). Then they were irradiated by a specified dose (0.5 Gy) to determine the reader correction factor (RCF). Finally, all the TLD chips were irradiated with a 0.5 Gy dose and their ECCs were determined. As it was predicted that the skin dose would be more than $1.0 \mathrm{~Gy}$, the calibration dosimeters were irradiated with $0.7,1.0,1.3,1.5,1.7$, and 2.0 Gy doses to plot the calibration curve. Based on the calibration curve, the TLD readings were corrected to compensate the supralinearity response of the TLDs. Seven TLD chips were located on individual patient's skin (Fig. 1), and measurement was performed during the irradiation of medial and lateral tangential fields (the TLDs were not on the skin when the supraclavicular region was being irradiated). Skin dose measurement was performed twice during two treatment sessions, and the mean dose received by each patient was calculated. Based on the data acquired for all the patients the mean dose at different points shown in Fig. 1 was also calculated.

\section{Statistical analysis}

A statistical software package for social sciences (SPSS, version 16.0) was employed to calculate the mean, maximum, and minimum skin doses received by each patient, all patients, and each point shown in Fig. 1. One-Sample Kolmogrov-Smirnov Test indicated that all the data had a normal distribution; therefore, the Repeated Measures Test was used to compare the mean dose of different points. The Bivariate Correlations Test was used to determine whether there is any correlation between the skin dose and beam entries separation, or thickness of the irradiated region. To analyse the difference between the skin doses of patients treated with and without wedge filter, Students' t-test was performed.

\section{Results}

The mean skin dose was averaged for all patients and also for those patients who were treated with and without wedge filter. The mean doses of points shown in Fig. 1 were also averaged for all patients. The results are presented in Table 1 . These results revealed that doses to points at middle and superolateral, and also doses to points at midlateral, superolateral, midmedial, and inferomedial are statistically equal $(p>0.05)$. However, differences between the first two points and the latter four points are statistically significant $(p<0.001)$. The mean dose to inferolateral is different from the other points $(p<0.05)$. Overall, the mean dose received by the lateral side of the irradiated region was more than the medial side $(p<0.001)$. The statistical analysis indicated that wedge filter caused a significant reduction in the mean skin dose $(p<0.05)$. The average difference between the treatments with and without wedge was 0.062 Gy per fraction (1.55 Gy for a complete irradiation of 50.0 Gy in twenty-five fractions to $(T V)$, which was completely originated from the points in the middle 
Table 1. Mean skin dose for all patients, patients who were treated with or without wedge filter, and average doses of individual points distributed on the skin, medial, and lateral sides of the irradiated region

\begin{tabular}{|lcccc}
\hline Parameter & Mean (Gy) & Minimum (Gy) & Maximum (Gy) & Number of patients/points ${ }^{\mathrm{a}}$ \\
\hline All patients & $1.466 \pm 0.089$ & 1.26 & 1.68 & 40 \\
\hline With wedge & $1.426 \pm 0.057$ & 1.32 & 1.54 & 14 \\
Without wedge & $1.488 \pm 0.095$ & 1.26 & 1.68 & 26 \\
Point middle & $1.630 \pm 0.167$ & 1.22 & 2.06 & 74 \\
\hline Point midmedial & $1.362 \pm 0.118$ & 1.07 & 1.68 & 70 \\
\hline Point midlateral & $1.410 \pm 0.145$ & 1.08 & 1.81 & 72 \\
\hline Point superomedial & $1.383 \pm 0.126$ & 1.17 & 1.73 & 70 \\
\hline Point superolateral & $1.628 \pm 0.181$ & 1.11 & 2.00 & 70 \\
\hline Point inferomedial & $1.373 \pm 0.144$ & 1.16 & 1.98 & 71 \\
\hline Point inferolateral & $1.491 \pm 0.210$ & 1.09 & 2.01 & 72 \\
\hline Mean dose of the points on the medial side & $1.403 \pm 0.119$ & 1.07 & 1.98 & 209 \\
\hline Mean dose of the points on the lateral side & $1.548 \pm 0.209$ & 1.08 & 2.01 & 217
\end{tabular}

a For the first three rows: number of patients; for the other rows: number of points

section (middle, midmedial, and midlateral). This is due to the fact that the mean doses of points at superomedial, superolateral, inferomedial, and inferolateral (the data are not presented herein) were equal for the with and without wedge filter groups $(p>0.05)$.

Figure 2 shows the mean skin dose of the individual patients evaluated (1.26 Gy $\leq$ mean dose $\leq 1.68$ Gy). For twenty patients the dose delivered to a point out of the medial field border was measured, and the results showed a mean dose of 0.182 Gy per fraction.

Bivariate correlations analysis showed that the beam entry separation (breast separation on the central axis) and thickness of the irradiated region have no effect on the mean skin dose $(R<0.289, p>0.05)$.

\section{Discussion}

In this study the skin dose of patients who underwent breast radiotherapy with and without wedge filter was measured. The effect of beam incident angle, the thickness of irradiated region (the thickness of the chest wall), and beam entry separation on skin dose was also analysed.

For delivering a 50.0 Gy dose to CTV the skin dose, averaged for all patients, was 36.65 Gy. The corresponding values for the patients who were treated with and without wedge filter were 35.65 and $37.20 \mathrm{~Gy}$, respectively. These values are adequate for patients with stages I or II cancers, for whom the skin involvement or recurrence is not a major concern. On the other hand, these results indicate that patients with stages III or more do not receive sufficient skin dose to prevent tumour recurrence; therefore, application of bolus material in part of the treatment course is necessary to increase their skin dose. Application of a bolus is more important for patients either with stage III or higher for whom a tissue expander is used, or for those who have a curved chest wall. This is due to the fact that these patients are mostly treated with a wedge filter, and

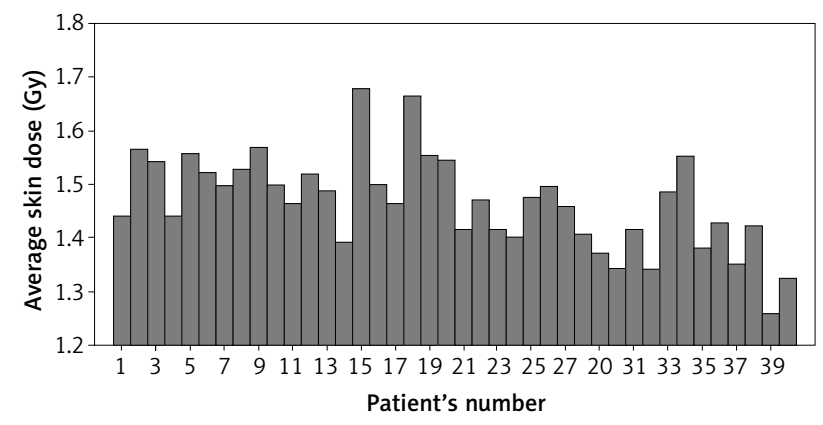

Fig. 2. Mean skin dose received by individual patients

results showed the average dose reduction due to application of wedge filter was 1.55 Gy during a complete treatment course (50.0 Gy to CTV in twenty-five fractions). Hsu et al. observed that a $2 \mathrm{~mm}$ thick Aquaplast, located on the chest wall, increased the skin dose up to $82 \%$ [8]. Andic et al. showed that a $1 \mathrm{~cm}$ thick bolus applied in 15 fractions of the total 25 fractions increased the minimum skin dose, while the increased maximum dose was tolerable [10]. Immobilisation casts are often used in breast radiotherapy to provide patient positioning reproducibility and limit the presence of skin folds. Kelly et al. observed that the application of an immobilising cast increased the skin dose up to $45.7 \%$ and $62.3 \%$, as measured with Gafchromic EBT film and MOSFT dosimeters, respectively [3]. These results indicate that the use of bolus or immobilising casts can compensate insufficient skin dose.

Cheung et al. [7] used GafChromic film to measure the skin dose to patients who underwent breast radiotherapy. Their results indicate for a target volume dose of 2.0 Gy in one fraction, the dose to the skin ranged from 0.87 to $1.06 \mathrm{~Gy}$, which is less than the amounts measured in the present study. The difference is mainly due to the different types of dosimeters used in the two studies. Based on the percentage depth dose distribution plotted for high-energy $\mathrm{X}$-rays, and based on a study carried out by Kron 
et al. [11], the dose received by skin is a function of depth in the skin layers. Therefore, GafChromic films, which are thinner than TLD chips, measure the dose received by skin epidermis, while TLD chips used in this study, with $0.9 \mathrm{~mm}$ thickness, measure the dose to dermis layer.

The statistical analysis revealed that the skin dose received by the lateral side of the treatment region was more than that received by the medial side $(p<0.001)$. This difference may be due to the different entrance doses of the lateral and medial beams, since the surface dose from the entry beam depends on the beam's angle [1, 4, 7]. The average incident angles of the lateral and medial fields were 51.0 and 34.2 degrees, respectively. Therefore, a larger entrance dose is expected for the lateral beam, and it causes a higher skin dose for the lateral side. The skin dose measured in the middle point (Fig. 1) was equal to dose received by the inferolateral point; however, it was higher than other points. The reason for this effect can be attributed again to the beam angle. Obviously, the incident angles of the lateral and medial beams with the middle point (the apex of the treatment region) are more than the beams' angles with the other points on the skin. Therefore, a larger skin dose is expected for the middle point. This finding supports the results reported by the Quach et al. study [4]. They applied a hemi-cylindrical solid water phantom to simulate the post-mastectomy breast treatment. They noticed that the surface dose was a function of beam angle incidence and was higher for tangential beams at the apex of the phantom.

Based on the results of the present study, there was no correlation between the mean skin dose and beam entry separation. This result is in contrast with other studies [2, $7,12]$. Cheung et al. found out that the skin dose was lower for large sized breasts [7]. However, those reported by Pires et al. referred to a study that revealed results in contrast with the results of Cheung et al.: "large breasts receive higher irradiation dose on the skin to assure adequate dosage in deeper structures and tissues" [2]. Roa et al. suggested that patients with large breasts may experience little skin sparing [12]. To interpret the lack of correlation between the skin dose and breast separation, observed in this study, the skin doses from the entrance and exit beams (the entrance and exit doses) should be analysed separately. Although large breasts (with large beam entry separation) cause a higher entrance dose needed to provide adequate dose for deep structures, they also result to a small exit dose, as radiation is attenuated to a higher extent in the thicker irradiated tissue. It has been proven that the contribution of the exit dose in the skin dose is more than the contribution of the entrance dose [7], which is consistent with our interpretation.

In conclusion, application of bolus material for postmastectomy advanced breast radiotherapy (for stages III and more) is necessary because the skin dose measured in this study was not adequate to prevent tumour recurrence. Among the factors whose effects on skin dose were evaluated, only beam angle affected the skin dose. The results revealed that the thickness of the irradiated region and the beam entry separation had no effect on the skin dose.
The authors declare no conflict of interest.

The authors would like to thank the office of the Vice-President for Research of Mashhad University of Medical Sciences for funding this work.

\section{References}

1. Khan FM. The physics of radiation therapy. 4th edition. Lippincott Williams \& Wilkins, Philadelphia 2010.

2. Pires AM, Segreto RA, Segreto HR. RTOG criteria to evaluate acute skin reaction and its risk factors in patients with breast cancer submitted to radiotherapy. Rev Lat Am Enfermagem 2008; ;16: 844-9.

3. Kelly A, Hardcastle N, Metcalfe P, et al. Surface dosimetry for breast radiotherapy in the presence of immobilization cast material. Phys Med Biol 2011; 56: 1001-13.

4. Quach K, Morales J, Butson M, Rosenfeld AB, Metcalfe PE. Measurement of radiotherapy $X$-ray skin dose on a chest wall phantom. Med Phys 2000; 27: 1676-80.

5. Yang J, Tong S, Li J. Skin dose determination for photon beam breast IMRT patients using Monte Carlo simulations and TLD measurements, 50th AAPM annual meeting, 27-31 July 2008, Houston, Texas, USA.s, USA.

6. Fogg P, Das K, Kron T, Fox C, Chua B, Hagekyriakou J. Thermoluminescence dosimetry for skin dose assessment during intraoperative radiotherapy for early breast cancer. Australas Phys Eng Sci Med 2010; 33: 211-4.

7. Cheung T, Butson MJ, Yu PK. Multilayer Gafchromic film detectors for breast skin dose determination in vivo. Phys Med Biol 2002; 47: 31-7.

8. Hsu SH, Roberson PL, Chen Y, Marsh RB, Pierce LJ, Moran JM. Assessment of skin dose for breast chest wall radiotherapy as a function of bolus material. Phys Med Biol 2008; 53: 2593-606.

9. Soleymanifard S, Bahreyni Toossi MT, Khosroabadi M, Vejdani Noghreiyan A, Shahidsales S, Tabrizi FV. Assessment of skin dose modification caused by application of immobilizing cast in head and neck radiotherapy. Australas Phys Eng Sci Med 2014; 37: 535-40.

10. Andic F, Ors Y, Davutoglu R, Baz Cifcis, Ispir EB, Erturk ME. Evaluation of skin dose associated with different frequencies of bolus applications in post-mastectomy three-dimensional conformal radiotherapy. J Exp Clin Cancer Res 2009; 28: 41.

11. Kron T, Elliot A, Wong T, Showell G, Clubb B, Metcalfe P. X-ray surface dose measurements using TLD extrapolation. Med Phys 1993; 20: 703-11.

12. Roa D, Chung R, Ramsinghani N, Al-Ghazi M. SU-FF-T-104: Simultaneous boost and skin dose toxicity reduction for breast cancer treatments using IMRT and RapidArc. Med Phys 2009; 36: 2543-4.

\section{Address for correspondence}

\section{Seyed Amir Aledavood}

Department of Radiation Oncology

Omid Hospital

Kouhsangi Street, Aalandasht Square

Mashhad, Iran

tel. +985138426082

fax +985138428622

e-mail: aledavooda@mums.ac.ir

Submitted: 29.01.2015

Accepted: 11.08 .2015 